\title{
ON THE COEXISTENCE OF EPILEPTIC SEIZURES AND ABNORMAL INVOLUNTARY MOVEMENTS*
}

\author{
BY
}

\author{
JERZY CHOROBSKI and TADEUSZ BACIA
}

From the Department of Neurosurgery, Academy of Medicine, Warsaw, Poland

Merely to nerve oneself to dare to speak on even a few of the many aspects of epilepsy and its allied pathological conditions is a brave venture, but to face such an assembly as this might surely be considered a sign of overconfidence. However, we, the pupils of the men taught by Charles E. BrownSequard, John Hughlings Jackson, William Gowers, and other neurologists of a similar calibre, feel it our duty to try to hobble along the path they walked so masterfully, and to present for analysis and judgment the fruits of our own modest endeavours.

This is indeed an appropriate time, as we are celebrating the centenary of "Queen Square", the Mecca of all neurologists, and the place of work of John Hughlings Jackson (1835-1911), undoubtedly the originator of much of our contemporary neurological thought on the epilepsies in particular. He had, as is well known, extraordinary foresight, for his many hypotheses have been fully verified by modern means of investigation.

Our contribution to Jackson's masterpiece on the problem of epilepsy is based on some six clinical observations. These raise questions which we should like you to help us to answer. The important question is: Are the abnormal involuntary movements, be they tremor or some choreo-athetoid movement, on the one hand, and, on the other, epileptic seizures, both of which occur in the same individual, caused by a single pathogenic focus, or are these two abnormal motor phenomena produced by two lesions separately located?

One aim of this communication is to summarize briefly three of six case histories and to present the relevant E.E.G. and E.C.G. findings, so that you may analyse them and draw from them some possible conclusions. However, it is not very surprising that we feel unable to refrain from trying to answer the question ourselves. In order that our answer, presented here in the form of a hypothesis,

\footnotetext{
* A paper read at the centenary celebrations of the National Hospital, Queen Square London for Nervous Diseases in June,
1960 .
}

may seem to be based on a firm foundation it is necessary to give a brief account of some of the ideas on the subject previously held by other investigators.

There is indeed "nothing new under the sun", for the above questions were posed in one form or another in many of Jackson's works on epilepsy, but we believe that they were not clearly answered. We remember, of course, that he considered the "epilepsies" to be a paroxysm dependent on a sudden temporary excessive discharge from some highly unstable region of the cerebral cortex. There is, in other words, if we follow Jackson aright, in each variety of epilepsy a "discharging lesion of some region of the cerebral cortex", and this is "nothing more than a group of cells whose instability is raised far above normal".

The same may be said about another variety of, to use Jackson's nomenclature, the "hyperkineses", namely, chorea, for he writes "my investigations lead me to the 'inevitable' inference that the movements observed in chorea depend on unduly high instability of nerve cells". Although he speculated "that, anatomically, the lesion responsible for chorea was to be usually found in some convolutions of the motor area", he was ready to admit in $\mathbf{1 8 6 8}$ that neither the site of the lesion (such as plugging of arterioles) nor the pathological process leading to it were known. He stated that on the anatomy and pathology of chorea all of us have hypotheses only. Discussing the patho-physiology, he said that it was clear to him that the cells and fibres governing the muscles so "copiously" moving, as in chorea, cannot be grossly destroyed. On the other hand, "since the movements of these muscles are disorderly in association, and in succession, the nerve cells and fibres cannot be healthy. Therefore, wherever in chorea the lesion may be, and whatever may be its particular cause, it is clear that its abstract nature (that is, detached from the incidents of any particular case, to use, this time, Herbert Spencer's terminology) must be one betwixt loss of 
function leading to paralysis of motion, and healthy function allowing the orderly movements of health. "In short, nerve-tissue in chorea as well as in other varieties of hyperkineses is neither destroyed nor healthy-it is irritable." Jackson considered, therefore, chorea and convulsions to be movements resulting from discharges of the cerebral cortex. The literature on the coexistence of these syndromes, in which the chief manifestations are abnormal involuntary motor acts, be they in the form of epileptic seizures or choreo-athetosis, is large and its proper analysis is out of the question in the short time at our disposal. Let us, however, note that Russell in 1899 found, on examining some 5,000 case histories, an extraordinarily frequent coexistence of epilepsy and chorea. Hartmann and di Gaspero (1914) maintained the same in their article on epilepsy in Lewandowsky's Handbuch der Neurologie, published in 1914. This combination was noted by Foerster in 1921 and 1926, and he mentioned this opinion held in Bechterev's (cited by Foerster, 1926) Féré's (1890), Binswanger's (cited by Foerster, 1926), and Lewandowsky's (1914) works. In 1904 Foerster published his own observations on paroxysms of choreiform movements occurring both before and after epileptic seizures or as epileptic equivalents. According to him, they were "undoubtedly" caused by a lesion of a like nature. Yet, he added, that since the pathological changes observed in such cases, for instance by Stertz in 1921, were mainly located in the corpus striatum, the lesions responsible for the epileptic seizures must be looked for elsewhere than in the basal ganglia. As the epileptic fits cannot be of "striatal origin" it must be inferred that the two sets of involuntary movements result from two separate lesions.

One recalls that in the years following the great epidemics of lethargic encephalitis Parkinsonism was common. There were also many clinical reports describing seemingly new types of fits, such as "extra-pyramidal" by Sterling in 1924 and by Wimmer in 1925, "striatal" or "subcortical" by Spiller in 1927, and "spastic" by Knapp in 1919. Wilson in 1940 pointed out that this conception was no novelty, except the names, because cases of that kind had long been known to Collier (1928) and to Wilson himself. The discovery, as pointed out by the last author, of striatal lesions post-mortem by Stertz (1921) is, of course, no proof of the striatal origin of the fits. On the contrary, they most probably had an extrastriatal source, since these ganglia were the seat of destructive processes.

Here, we must add a few words about the far from rare combination of myoclonus with epilepsy, known as Unverricht-Lundborg's myoclonic epilepsy. The Lundborg (1912) type asserts itself as a myoclonic aura of a major epileptic seizure, or as its moto equivalent. The so-called second type of this syno drome, the continuous myoclonus of Kojewnikow? seems to be a sequel of a local inflammatory process? It is interesting to note that extirpation of the cortero involved abolishes the local hyperkinesis, as reporte by Grinker in 1937. The third type, progressiv familial myoclonus of Unverricht (1891), begins wit a generalized attack; it is only rarely that myoclonic. jerkings occur at the start of the disease. Unfortuns ately, nothing is known about the patho-physiologश of this most interesting syndrome, so we end this paragraph by quoting Wilson, who in 1940 said thas it "does not differ in any essential feature from the clinical commonplace of fits combined with jerks"

Now, returning to the problem of strict localis zation of this or that type of epilepsy, let us recal $\mathbb{P}$ that though Jackson used the term striatal epilepsy $\vec{\omega}$ he also used such phrases as "unilateral convulsion" "hemiplegic epilepsy" or "convulsions beginning unilaterally". But is it not true, as has been so we expressed in 1953 by William G. Lennox, one of the most eminent contemporary students of epilepsyic that Jackson's greatest achievement is not his description of a certain seizure pattern but his formulation of concepts or principles that explaip seizures of all sorts.

What interested Jackson was the study of the "morbid alteration of the normal function of neve tissue" and the need to understand the function of the brain in health. Jackson asks what is function of the healthy nervous system. It is t\& store up and expend force. It is the function of nerve units, in expending their stored up force, $t{ }^{2}$ develop certain more or less complex movements, ind correspondence with special, not always constant excitations which bring the local movements into harmony with the whole organism. And finallis there are, for him, two kinds of alterations of function due to disease: loss of function and overe function. In the latter, more nerve force is stored up than in health and more is therefore expended; the nerve tissue is highly unstable. Under the firs? heading come palsies, akineses, anaesthesiae; unde? the second chorea, the epilepsies, tetanus, the hyperkineses, neuralgia, etc. In a footnote he added to the above-quoted words a very significant remark, namely, that "the functional change of nerve tissue in epilepsies, in chorea and in tetanus is supposed to be the same".

All this is not only of great interest for anybodf studying the history of Jackson's neurological ideasin but of very special value to our own approach to th\& problem of the pathomechanism of the hyperkineses. We will shortly show why this is so, but first let us: give a very brief account of our own observations. 
We have had under our observation six patients in whom epileptic seizures were concomitant with choreo-athetoid or simply athetoid movements. Two of them have been operated upon, and in another the brain has been examined by means of ventriculography. In these three patients we were able to demonstrate that those two syndromes may be caused apparently by two differently located lesions. The one responsible for abnormal involuntary movements is probably of a special, most often, diffuse nature. This had been proven to our satisfaction by the facts, first that the excision of the motor cortex, made in order to relieve the patient of his choreo-athetoid movements, actually brought about cessation of these movements while the epileptic seizures continued, and secondly that not until the pertinent epileptogenic focus, localized by means of E.E.G., E.C.G., and by electrical stimulation in another region of the brain than the motor cortex, had been removed did the epileptic fits cease.

The excision of a relevant area of the motor cortex, a procedure used by Horsley (1909) and Bucy (1942) in cases of abnormal involuntary movements of all kinds, is a palliative operation. The rationale for its use is that by attacking a strategic point in the pyramidal and extrapyramidal nervous systems it is possible to interrupt a vicious circle of pathological nervous impulses causing these abnormal movements. We will return to this later.

Obviously, when the epileptogenic focus happens to be located in or around the motor cortex itself, then the excision of that cortex is followed by cessation of both the epileptic seizures and the choreo-athetoid movements. This has been observed in the second of our patients. We may consider, therefore, the results in this second patient most important in our study of the problem, especially when we compare the results with those obtained in the first patient.

The case histories of these two patients will be given in some detail, as well as that of a third patient. This last case has been added because it presented an exceptionally interesting phenomenon, namely, complete cessation first of the athetoid movements, then of the epileptic seizures, both following a simple ventriculography.

\section{Case Reports}

Case 1.-B.T., a girl, aged 15 years, when 3 years old, suffered severely from whooping-cough, during which she started to have generalized epileptic fits, with a visceral aura, loss of consciousness, and clonic movements of the right hand. At first the fits occurred three times a year, and recently several times a week. The reason for her admission was a severe status epilepticus. After the first epileptic attack the child lost her speech 5 and became paralysed down the right side. Recovery took place to some extent, but her right hand, weak and spastic, became the seat of typical athetoid movements. The girl was neuropathic and mentally retarded. Ventriculography showed only a dilatation of the left lateral ventricle including the temporal horn. The E.E.G. tracings, taken before her first operation, indicated that we were dealing here with two aspects of abnormal electrical activity. First there were episodes of spikes and slow waves located in both temporal lobes, and secondly diminished voltage, suggesting diffuse cerebral damage.

After having ascertained at operation the exact position of area 4, especially that part controlling movements of the upper extremity, we sucked out that portion subpially on April 18, 1958. We did not touch the temporal lobe, which was macroscopically apparently normal. In the electrocorticogram, however, prominent spikes and slow wave complexes were picked up from over the temporal lobe. As expected the athetoid movements ceased, but improvement lasted only for some three months, when some abnormal movements returned. She continued throughout to have epileptic seizures, and the E.E.G. examination confirmed the presence in the left temporal lobe of a focus of frequent spikes and slow waves. It is true that the spikes were now less frequent than before the operation and were of lower voltage, but they still were there.

She was, therefore, operated upon for the second time on November 4, 1958. Because electrical stimulation of the cortex, close to the previously sucked-out area, was yielding definite movements of the right fingers and the hand and she still had some athetoid jerks, it seemed that we had not removed sufficient of area 4 for her hand movements. We therefore sucked out more cortex from area 4. It was our hope that not only would we relieve the patient of the athetoid movements completely, but that perhaps this added excision of the motor cortex would also influence the epileptic seizures. As the E.E.G. and electrocorticogram from over the left temporal lobe was less abnormal than previously, the lobe was not excised. After this operation the athetoid movements ceased completely but the epileptic fits continued to torment the patient.

We submitted her, therefore, one year later, on December 18, 1959, to a third operation, during which we produced by electrical stimulation of the upper temporal region her typical epileptic seizure, thus confirming the presence of an epileptogenic focus, which was only assumed from the E.E.G. and E.C.G. tracings. Consequently, we sucked out this area. Our hopes for improvement were fulfilled, for the patient finally became free of the epileptic fits. The E.E.G. from over the excised temporal area showed no spikes and slow wave complexes. It is true that only six months have elapsed since the last operation, but the patient continues to be well. We must add that before the operation full medical anticonvulsant treatment had been of no avail.

Following presumably an acute virus encephalitis at the age of 3 , the girl, after a short period of complete loss of speech and right-sided spastic 
paralysis, developed generalized epileptic seizures, starting in the right hand, and also athetoid movements of the right hand and fingers. Removal of the relevant motor cortex relieved the patient of the athetoid movements, whereas the epileptic fits only ceased after excision of the epileptogenic focus, which was located, by means of E.E.G. and E.C.G. tracings and electrical stimulation, in the left temporal lobe. Later E.E.G. examination showed, however, that the slight but diffuse abnormal electrical activity was not confined to the temporal lobe, but was evident over both sides of the brain, presumably being the result of diffuse damage by the whooping-cough infection.

We believe, and will try to explain more fully later on, the reasons for our belief that the pathomechanism of this patient's athetoid movements is to be looked for in a disturbance in the dynamics of the nervous pathways, the integrity of which is necessary for the performance of any normal motor act. We believe that this disturbance was caused by the diffuse damage to the brain. The epileptic seizures, on the other hand, must have been produced by a lesion, located in the temporal lobe. What the nature of this lesion may be, we do not know.

Case 2.-M.W., a girl, aged 11 years, without any apparent cause, had developed, at the age of 11 months, left-sided epileptic seizures, starting in the left hand. After this, she developed a spastic hemiplegia, and choreoathetoid movements in the left hand and fingers. To these left-sided epileptic fits beginning unilaterally and occurring once or twice a month, there were added, at the age of 4 years, peculiar attacks about once a month, characterized by a laryngeal and oesophageal spasm, reddening of the face, a loud shriek, jerking of the left extremities, without loss of consciousness.

Electroencephalographic tracings, typical for all cases of that or the other type of abnormal involuntary movement, favoured a diffuse pathology. There were present so-called slow spikes. An electrocorticogram done at operation on March 5, 1958, showed an area of abnormality more evident over the right motor gyrus, close to the first temporal convolution. It was characterized by sharp waves, and two or three episodes of apposition of waves at nearby electrodes. This area corresponded to a spot in the precentral motor gyrus, the white matter of which was definitely tough and the leptomeninges covering it were whitish and thick. Electrical stimulation of this region proved it to be precisely the motor area for hand and finger movements. By slightly increasing the usually employed parameters of stimulation, we produced her typical epileptic seizure, i.e., one starting with flexion of the left hand and fingers. From no other region of the exposed cortex, not even from that in the closest vicinity of the recently stimulated area, and not even with strongest stimulation, were we able to produce a fit. Presumably this was an epileptogenic focus, and since this region corresponded exactly to area 4 for han $\mathbb{P}$ movements, we performed the Horsley-Bucy operation This was carried out on March 5, 1958, and as expecte from our previous experience of some 50 cases using the same procedure, we immediately and completely relievedo the child of the choreo-athetoid movements. The relief remained even after recovery from the transient hems plegia. The epileptic seizures continued for some eigh" months, in spite of continuous and heavy anticonvulsan treatment. An E.E.G. examination, made after pentothat. provocation, demonstrated in the left temporal lobe $\underset{P}{\stackrel{D}{P}}$ definite pathogenic focus which had not been seen ig the straight E.E.G. (Fig. 1). Then, though there was no change in treatment, the epileptic fits ceased, an have not reappeared during the 18 months in which the patient has been followed up.

This is, it seems to us, an important case. Thể epileptogenic focus was placed more or less exactl 9 in the motor area for hand movements, which wass macroscopically grossly abnormal. Its excision, donల్ to relieve the patient of her abnormal involuntar㐫 movements, freed her, therefore, both from the epileptic seizures and the choreo-athetoid moves ments. We have all been taught that a patienitu suffering from epileptic fits loses them the moment he becomes paralysed. We know, however, that this is not always true, and should the epileptogenic focus be localized elsewhere than in or about the metor region, than even a complete and long-lasting paralysis, caused by the excision of the motor cortex, does not necessarily mean cessation of epile seizures.

Case 3.-S.S., a man, aged 23 years, some six month요 after a head injury, followed by unconsciousness for tw weeks, began to have epileptic seizures, ushered in by aळ aura lasting for about half an hour, during which timg he felt suddenly weak and generally "bad", and ha\$ troublesome vertigo. The tonic-clonic, generalized fits of which he had had three in the seven months before his admission began with loss of consciousness, rotation of the head and body to the right, and jerking of the right hand This was followed by a temporary paresis. The E E.G. showed a slight bilateral and diffuse abnormalit without any characteristic details (Fig. 2). Since there was a history of a head injury and a purulent discharge particularly from the right ear, ventriculography was done on October 13,1958, to exclude an abscess. Ne abscess was found. It was most interesting that some 11 days after ventriculography the athetoid movements of his right hand, which had appeared some time afte? the injury, disappeared completely. There was also some slight improvement in the E.E.G., though the tracing were by no means normal.

It is true that during the patient's three-week stay in hospital we never observed an epileptic seizure but this seems to us of little signficance as he had had them so rarely anyway. However, the dis appearance of the abnormal involuntary movemente 


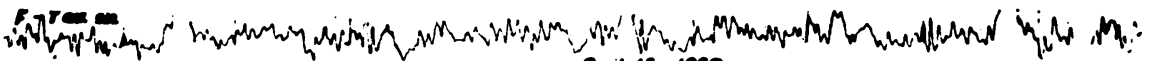

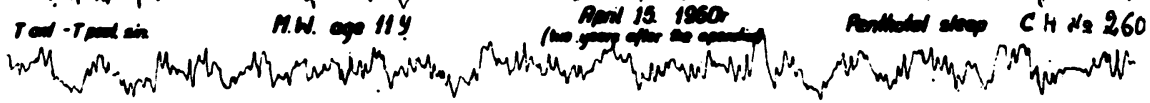

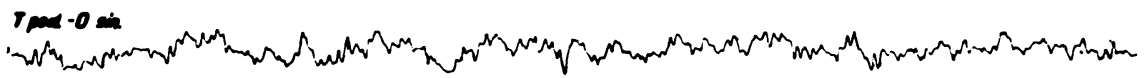

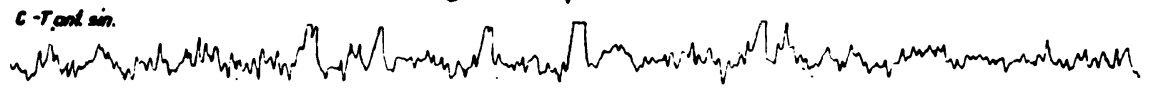

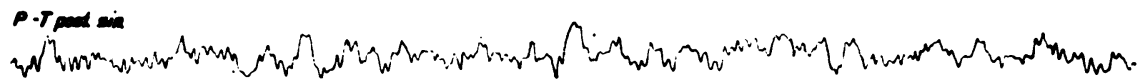
F. Tout. dex

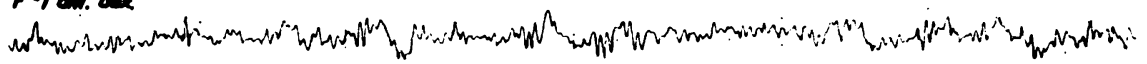

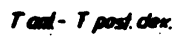

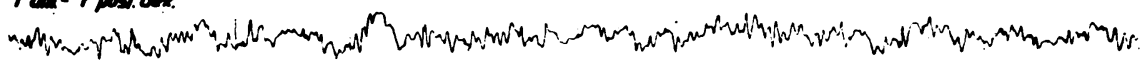

\section{Tpoud -0 ans.}

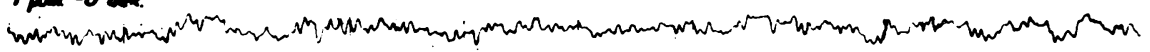
C-rancur.

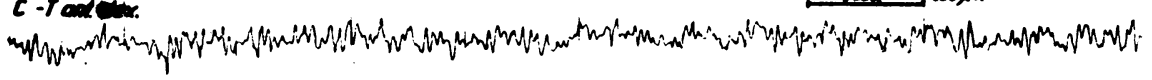

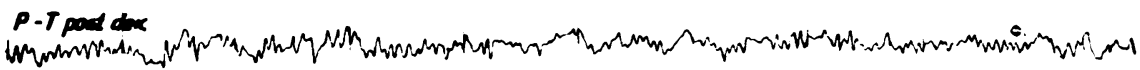

FIG. 1.-E.E.G. tracing (Case 2) demonstrates in the left temporal lobe a definite pathogenic focus not seen in the straight E.E.G.

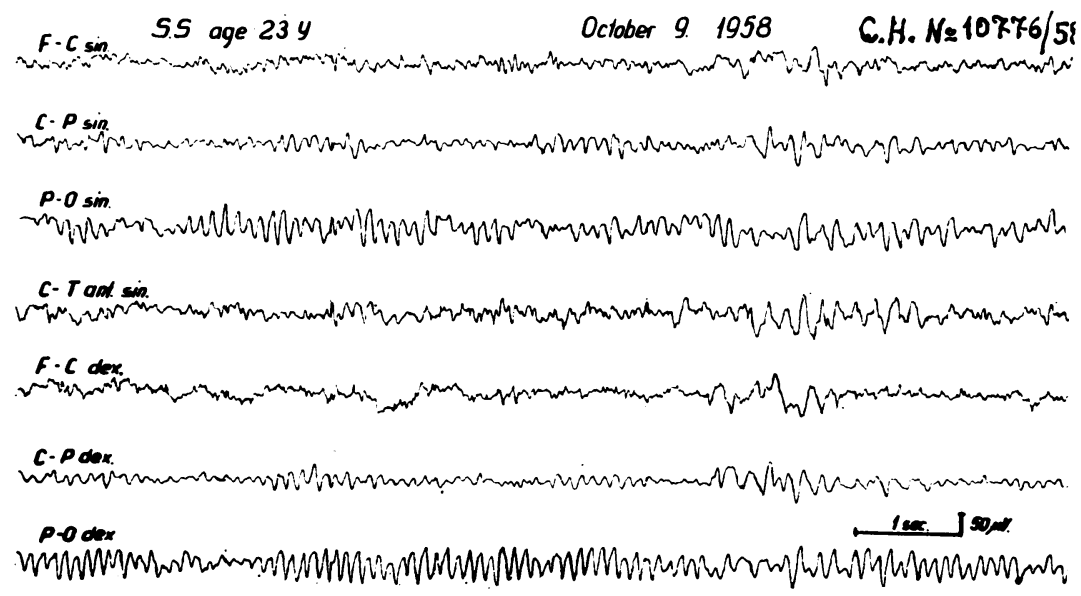

c.tod clex.

FIG. 2.-E.E.G. tracing (Case 3) shows a slight bilateral and diffuse abnormality without any characteristic details. This is an E.E.G. picture typical in cases of abnormal involuntary movements. 
is, at least, interesting though not entirely unexpected. We have observed them go, when caused by a brain tumour, after simply opening the dura mater. The implication of this certainly unusual phenomenon needs some comment later.

Allow us to add the following few words about the rest of our cases. As already mentioned, the E.E.G. and the E.C.G. tracings were in all of them abnormal. One of our tracings demonstrated this abnormality which is as a rule asymmetrical. In the CT right anterior tracing we see there a complete absence of the alpha rhythm, with some episodes of fast waves of relatively high voltage while on the left side the alpha waves are present.

Sometimes, no signs characteristic of epilepsy are to be found in the E.E.G., as in the case of a girl aged 13 years. On the other hand, there usually is some asymmetry in the tracings, pointing towards the site of, as it were, "epileptic readiness", and not to the site of the origin of the abnormal involuntary movements. Since neither operation nor ventriculography were carried out in those three cases, no further comment will be made, as it would not be of any value either for or against our hypothesis.

\section{General Comment and Conclusions}

We of course realize perfectly well that the cases presented here in some detail cannot serve as a foundation for truly binding conclusions, if for no other reason than because their number is too small. However, may they not serve as a pretext for further investigation? We hope that they may, since according to Jackson, "an hypothesis, otherwise a supposition, is not a conclusion but only the starting point for methodical observation and experiment, the endeavours being not only to prove it, but to disprove it".

We believe that diffuse damage to the brain, as commonly demonstrated by E.E.G. in patients suffering from the combination of the two syndromes under discussion, may result from injury, sustained at birth or later, infection, intoxication, or some other pathological factor. This diffuse damage, however, is not enough to provoke epilepsy. There always must be, and frequently we have been able to demonstrate its presence by modern means of investigation, somewhere in the brain a so-called epileptogenic focus. We knew its bio-electrical appearance, but to what kind of a lesion it actually corresponds, we do not know. It is true that Jasper in 1954 reminded us that for Jackson the basic physiological and chemical change underlying the initial discharge is probably the same in all cases, although the pathology may vary widely. Does this help us in the understanding of the nature of the harmful or epileptogenic focus?
We postulate, then, and after all our two cases seem to point to the reasonableness of such supposition, that in cases with epileptic seizures anc abnormal involuntary movements the first are caused by a separate pathogenic focus, localized is one or other region of the brain, while the abnormas involuntary movements are due to a diffuse involveâ) ment of the central nervous system (Chorobskis. 1957 and 1961).

Here, we can but summarize our ideas very? briefly. First, we draw your attention to the fact that intracranial tumours of any nature and locatior quite often provoke the appearance of "classical's" syndromes, commonly attributable to involvemen $\mathbb{R}$ of the extrapyramidal system. This view is based oफ़् personal observation in 14 cases and on reports in the literature. Contrary to what is generally observed in cases of abnormal involuntary move $\vec{\omega}$ ments associated with birth injury, infection, of intoxication of the brain, the abnormal involuntar movements caused by brain tumours disappear aftef the removal of their cause, even though no paresis of paralysis of the extremities is produced. Further, the improvement of the motor activity, observed in the brain tumour cases, is often accompanied by return of a more or less normal electrical activityof the brain. Such observations are of consideratie interest.

In four of these 14 cases, the abnormal involuntary movements were concomitant with epileptic seizunese The most important point, however, is that after the tumour has been removed the epileptic seizure may either disappear simultaneously with the involuntary movements or they may not, thus supporting our assumption that the pathomechanisnow of these two pathological phenomena is not necese sarily the same.

The cessation of the abnormal involuntary move 3 ments after the tumour has been removed, and everg following simple incision of the dura, i.e., a suddep? change in the intracranial pressure, seems to confirm our idea of a functional rather than an anato mical cause of these movements. It is postulated therefore, that the "derailment" of the innervatio normally necessary for the maintenance of propes muscle tone and for the performance of a norma motor action, or of both, is brought about by stimuli coming from so-called reverberating chains of neurones. These stimuli interfere with the smoot cooperation of the pyramidal and extrapyramida nervous systems, i.e., with the conditio sine qua non of any normal motor activity. This interferenceu takes place at the moment when the afferent nervous system is "busy" conducting the nervous impulse ${ }^{\kappa}$ necessary for starting and controlling that activity?

We believe that this harmful action of chains of 
neurons, located mainly within the plexiform layer of the cerebral cortex, or perhaps in the reticular formation of the brain-stem also, is brought about by their interfering with the nervous systems necessary for the performance of any motor action and may consist of an inopportune and/or inordinate emission of excitatory, and/or inhibitory stimuli which thus disorganize the proper maintenance of the muscle tone, and the performance of normal motor activity. These, as it were, extrasystemic stimuli may play upon some special nervous circuits, located in the midbrain, the malfunction of which results in, as some recent investigations tend to suggest, a Parkinsonian tremor. They may also act on the spinal gamma system which, of course, is responsible for muscle tone and is prerequisite for alpha system activity.

The cause which brings into play the reverberating neuronal chains, and produces the disturbance in the dynamics of the nervous system, may well be found in a case of brain tumour in the tumour's direct pressure upon the relevant nervous circuits or indirectly in the action of the tumour on them through the interference with the blood supply or oxygenation of the chains, intoxication of the brain by products of tumour metabolites. The same mechanism is probably at play in cases of abnormal involuntary movements of other aetiology.

And so, we come to the end of this communication which should be accepted as a kind of provocation to further study. We find some justification in suggesting this idea in view of Huxley's statement that the anticipation of Nature "by the invention of hypotheses even based on incomplete deductions ... . has proved itself to be a most efficient, indeed an indispensable instrument of scientific progress" (Science in the Reign of Queen Victoria, v.3).

\section{REFERENCES.}

Bucy, P. C. (1942) Res. Publ. Ass, nerv, ment, Dis, 21, 551.

Chorobski, J. (1957). Patomechanism i Leczenie Chirurgiczne Ruchow Mimowolnych. P.Z.W.L., Warsaw.

(1961). A.M.A.Arch. Neurol. Psychiat. To be published.

Collier, J. (1928). Lancet, 1, 587, 642, 687. Quoted by Wilson (1940). Féré, C. (1890). Les épilepsies et les épileptiques. Quoted by Foerster (1926). Alcan, Paris.

Foerster, O. (1921). Z. ges. Neurol. Psychiat., 73, 1.

- (1926). Disch. Z. Nervenheilk., 94, 15.

Grinker, R. R. (1937). Neurology, 2nd ed., p. 840. Thomas, Springfield, Illinois.

Hartmann, F., and Gaspero, H. di (1914). "Epilepsie" in Lewandowsky, M. Handbuch der Neurologie, Band 5. Spezielle Neurologie IV, p. 832. Springer, Berlin.

Horsley, V. (1909). Brit. med. J., 2, 125

Huxley, Th. H. In Selected Writings of John Hughlings Jackson, ed James Taylor, Vol. 2, p. 372. Staples Press, London (1959).

Jackson, J. Hughlings (1868). Edin. med. J., 14, 294.

Jasper H. (1954). In Penfield, W., and Jasper, H. Epilepsy and the Functional Anatomy of the Human Brain. Little, Brown, Boston.

Knapp, A. (1919). Mschr. Psychiat. Neurol., 46, 47. Quoted by Wilson (1940)

Lennox, W. G. (1953). In The Founders of Neurology, ed. Haymaker, W. E. Thomas, Springfield, Illinois.

Lewandowsky, M. (1914). Handbuch der Neurologie. Bd. 5. Spezielle Neurologie IV. Springer, Berlin.

Lundborg, H. (1912). Z. ges. Neurol. Psychiat., 9, 353.

Russell, J. W. (1899). Brain, 22, 593. Quoted by Hartmann and Gaspero.

Spiller, W. G. (1927). Brain, 50, 171. Quoted by Wilson (1940).

Sterling, W. (1924). Rev. neurol., 22, 484.

Stertz, G. (1921). Der extrapyramidale Symptomen-komplex. Karger, Berlin.

Univerricht, H. (1891). Die Myoclonie. Deuticke, Vienna.

Wilson, S. A. Kinnier (1940). Neurology, Vol. 2. Arnold, London. Wimmer, A. (1925). Rev. neurol., 21, 281. 